\title{
Morphological Changes Induced by Instant Noodles Food on Kidney of Adult New Zealand Rabbits
}

\author{
Eze, G.I ${ }^{1}$, Ehimigbai A.R.O ${ }^{1 *}$, Ezugwu, N.S ${ }^{1}$, Okafor, F.U ${ }^{2}$ \\ ${ }^{I}$ Department Of Anatomy, School Of Basic Medical Sciences, College Of Medical Sciences, \\ University Of Benin, Benin-City, Edo State, Nigeria. \\ ${ }^{2}$ Department Of Nursing, School Of Basic Medical Sciences, College Of Medical Sciences, University Of Benin, \\ Benin-City, Edo State, Nigeria.
}

\begin{abstract}
The morphological changes induced by instant noodles food on kidney was evaluated using adult newzealand rabbits. The study was carried out on twenty-two healthy male and female Rabbits, weighing between 0.7-1.5 $\mathrm{kg}$ for 63 days. While two rabbits died during the acclimatization period, the remaining rabbits were randomly assigned into four groups $A, B, C$ and $D$ and having five $(n=5)$ each. Group $A$, is a control fed with normal food only, group B fed with normal food and noodles only, group $C$ fed with normal food and seasoning only and group $D$ fed with normal food and noodles with seasoning. The morphological changes on the kidney of the rabbits was evaluated by histopathologic examination.

Histologically, Group A showed normal glomeruli, tubule and vasculatures of the kidney along with normal inner lining of the blood vessels.

Group B showed moderate interstitial congestion with chronic inflammation that is perivascular. The group $C$ shows ulceration of the intima.

Group D shows deposition of hyallinised materials inside the wall of the blood vessels and also, there is narrowing of the lumen of the blood vessels and deposition of the hyalinised materials.

Finally, consumption of instant noodles may lead to pathological changes in the kidney.

Keywords: Morphological changes,Induced,Instant-noodles, Kidney, New Zealand rabbits
\end{abstract}

\section{Introduction}

Instant noodle package is composed of noodles, pepper and seasoning powder. They are made instant by frying (Crosbieetal.,1998). Noodle has been known extensively in our locality and globally reported to have caused some health hazards, including gastrointestinal tract (GIT) disorders, increased intraocular pressure (Inagaki, 1996). Noodles are typically fried as part of the manufacturing processes (Hou, 2001), resulting in a higher levels of saturated fat. Wheat, flour and salt are mixed together followed by massing and the dough is rolled flat into sheet and cut (Hou, 2001). After processing, they are just packed and sealed, living the elements out (Hou, 2001).

Instant noodle and it's flavoring sauce base contain high amount of monosodium glutamate (MSG), which causes neuroplacental neurotoxic effect (Gao, et al., 1994), causes cataract, causes induced retinal lesions (Chambille, et al., 1993) and causes genotoxicity (Faronmbi, et al., 2006). Another concern regarding the consumption of fried foods like the instant noodles is the possible presence of oxidation products resulting from poor maintenance of the oil (Kubomura, 1998). Various health hazards have been revelation of it's consumption (Inagaki, 1996). The ingredient (seasoning) has monosodium glutamate (MSG), which is a flavor enhancing food additive (Morris et al., 2000). This could increase the possibility of it's inadvertent consumption in high concentration. The instant noodles manufacturing process usually make use of the application of various substances like sodium, oxidized fat and oil (during instant frying) which might be detrimental to health (Yun $e t$ al., 1996). Monosodium glutamate has been reported to have caused various health hazard, including gastrointestinal tract disorder (Eweka and Om'Iniabohs, 2007), damage to arcuate nucleus of the brain (Belluardoet al., 1990). of a rabbits

The aim of the study is to determine the effect of excess consumption of instant noodles on the kidney

\section{Materials And Methods}

Experimental Animals and Management

The study was carried out on twenty-one healthy male and female Rabbits, weighing between 0.7-1.5 $\mathrm{kg}$. The Rabbits were obtained from a livestock farm in Ikhin town, in Owan east Local Government Area of Edo State. The animals were brought to animal house of the department of Biochemistry, University of Benin, Benin City, where they were kept in metallic cages. All animal experiments were conducted in compliance with the humane animal care standards outlined in the 'Guide to the care and use of Animals in Research and 
Teaching' as approved by the Institute of Laboratory Animals Resources National Research Council, DHHS,Pub. No. NIH 86-123 (1985). The animals were allowed to acclimatize for three weeks, under standard laboratory conditions of temperature, humidity and illumination (of 12 hours dark and 12 hours light). They were fed with stock growers, an elephant grass, which serve as a source of roughage for the Rabbits and water ad libitum. During three weeks of acclimatization, four rabbits died. After the acclimatization, the remaining seventeen rabbits were randomly assigned into four groups A, B, C and D, with A, B, and C having (n=4) each, and $D$ having $(n=5)$. The duration of the experiment was nine weeks and two rabbits died on the process. The control group A, was fed with elephant grass, feed mash and water ad libitum only throughout the period of the experiment. The other group B, C and D were fed with instant noodle pack, gotten from the Hostel shopping complex, beside June 12 complex building, University of Benin, Benin City .

\section{Instant Noodle Pack Administration}

Group A is a control, fed with normal food only (Elephant grass and Feed marsh). Group B received 1 packet of noodle daily (about 120g) and normal food. Group C received 1 sachet of the seasoning powder with the pepper and normal food every day. Group D received 1 packet of noodle (about $120 \mathrm{~g}$ ) plus 1 sachet of the seasoning powder with the pepper and normal food every day.

Histology

After sixty-three (63) days of administration, the animals were sacrificed by cervical dislocation. With the aid of surgical blade and forceps, the kidneys were harvested, the harvested kidney tissues were put in universal specimen bottles, filled with $10 \%$ formal saline for fixation for 48 hours.

After 48 hours of fixation, the fixed tissues were processed using the paraffin wax embedding technique, after which the slides were examined by the aid of an Olympus microscope and the images subsequently converted to micrographs by the use of a digital microscope.

\section{Results}

Micrograph And Interpretation

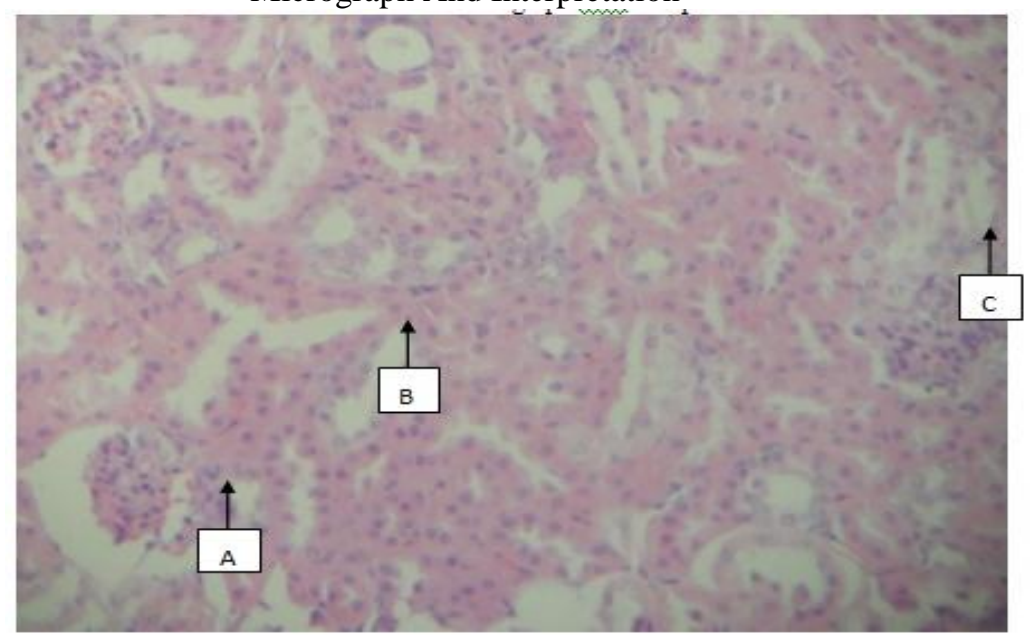

Plate 1: Control Kidney showing glomeruli A, tubules B andinterstitial spaces C (H\&E x 10)

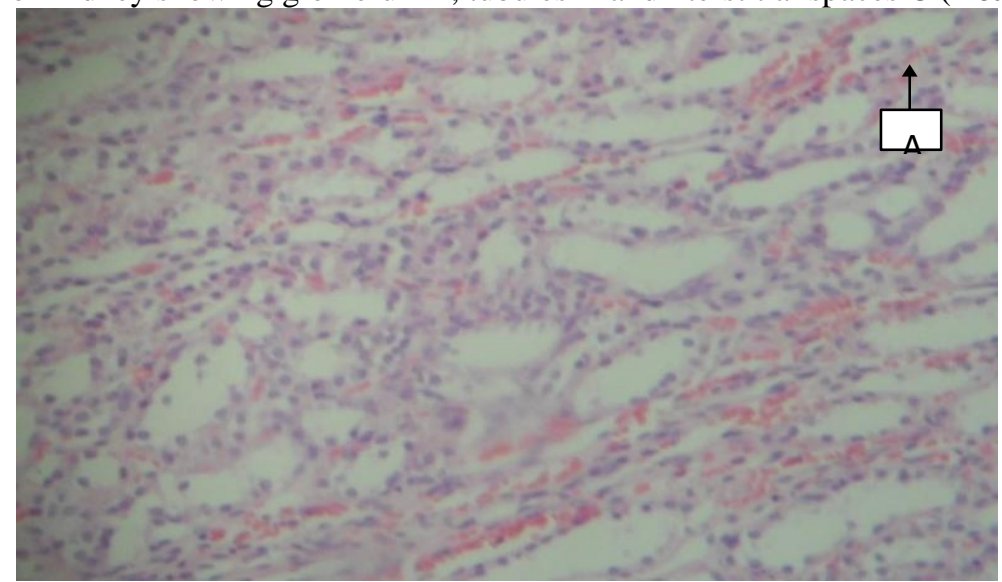

Plate 2: Rabbit Kidney treated with noodles and feed showing moderate interstitial congestion A (H\&E x 10) 


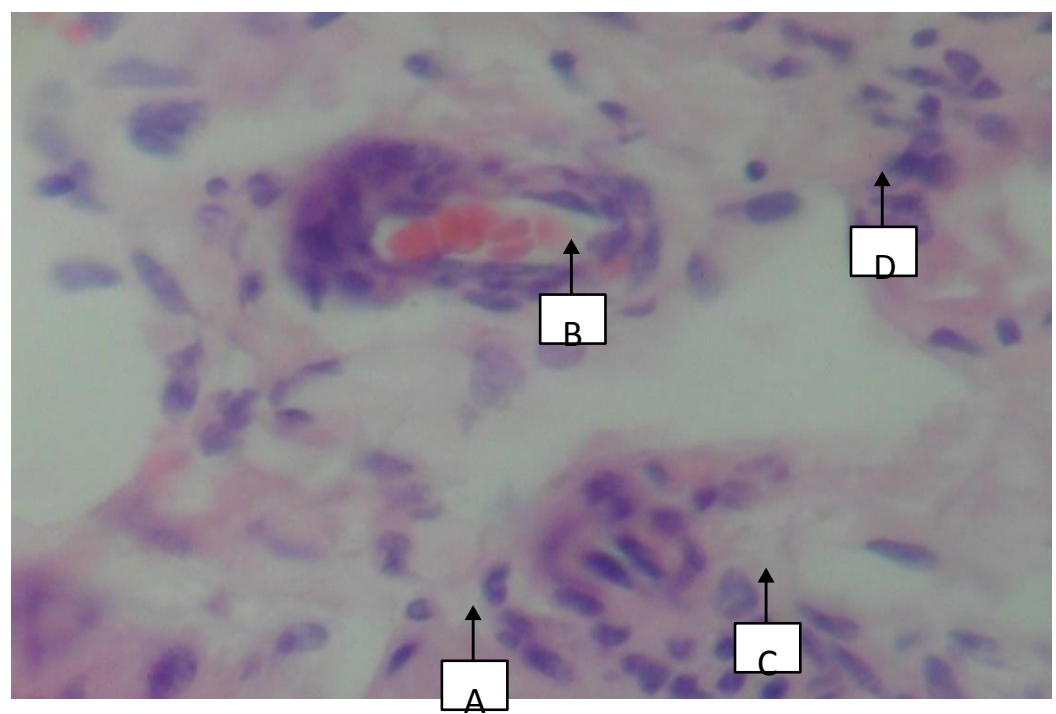

Plate 3: Rabbit Kidney treated with noodles and feed showing mild interstitial oedema A, vascular congestion and hypertrophy $B$, mild interstitial fibrosis $C$ and mild infiltrates of chronic inflammatory cells D (H\&E x 40)

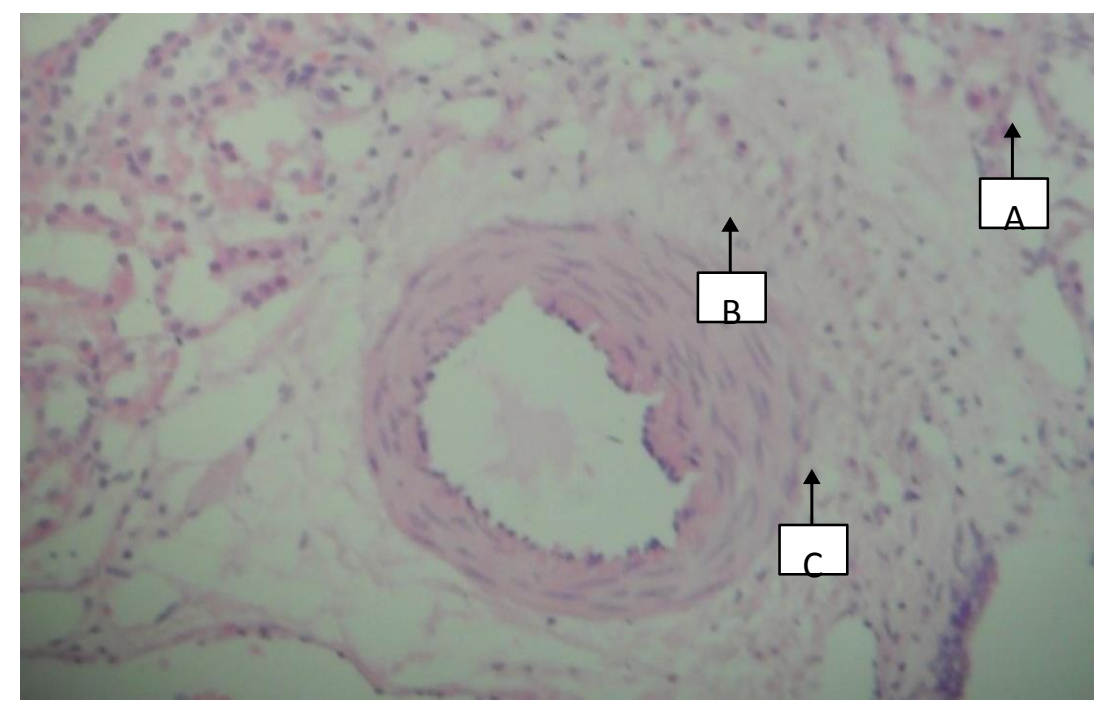

Plate 4: Rabbit Kidney treated with seasoning and feed showing mild interstitial oedemaA, moderate vascular hypertrophy $B$ and patchy vascular intimal ulceration C (H\&E x 10)

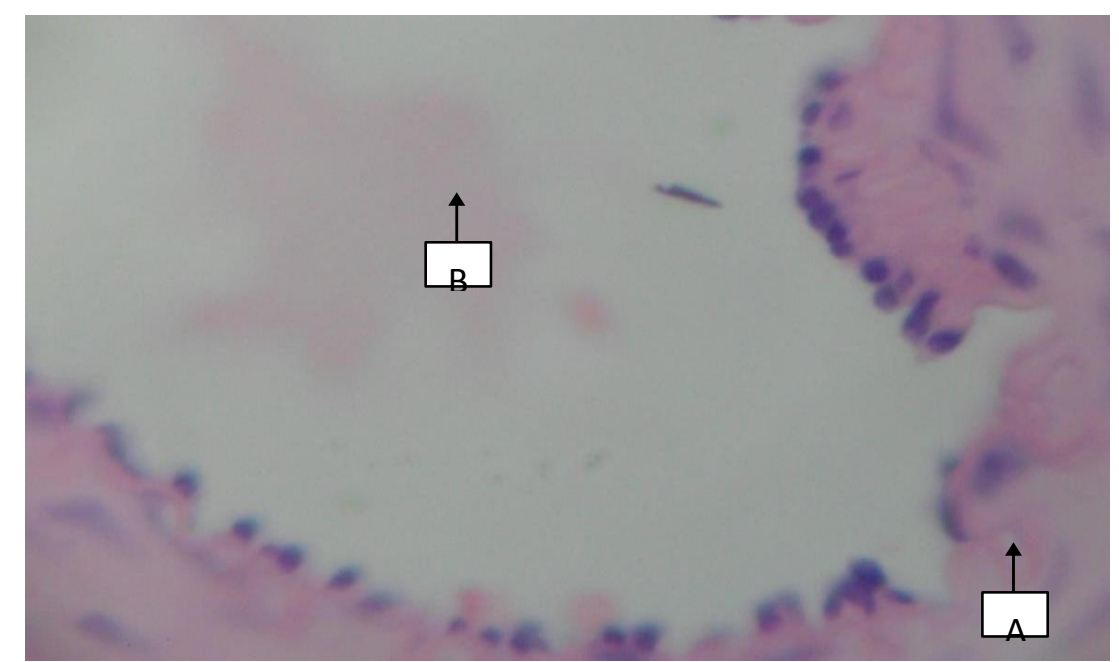

Plate 5:Higher power of the lesion above showing intimal ulceration A and hyalinised material B (H\&E x 40) 


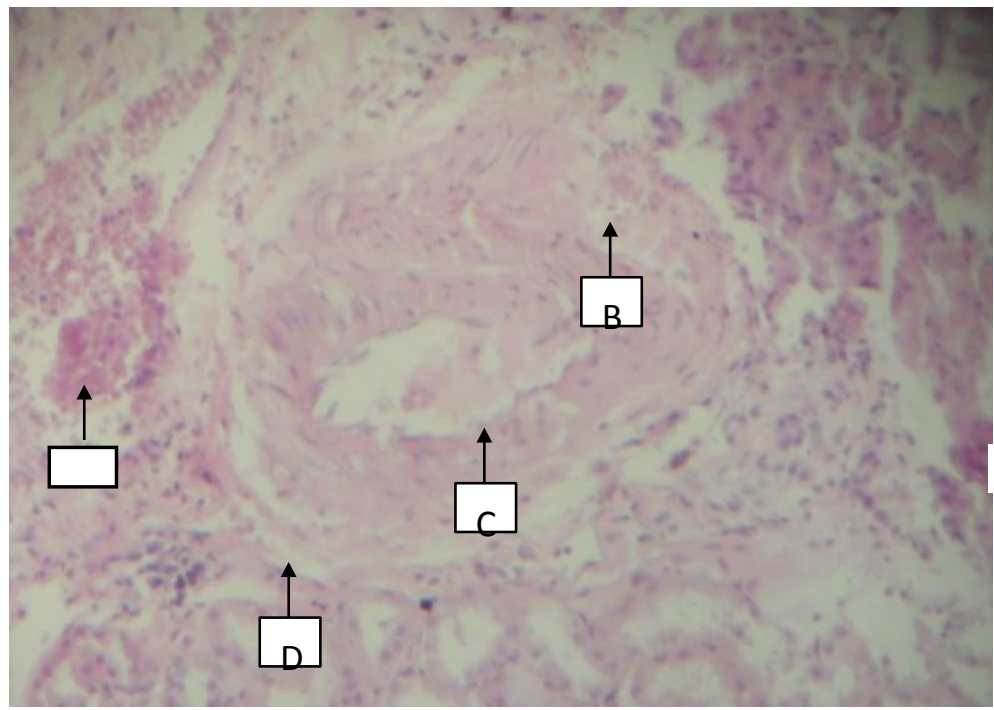

Plate 6: Rabbit Kidney treated with noodles, seasoning and feed showing moderate interstitial oedema A, moderate vascular hypertrophy $\mathrm{B}$, intimal ulceration $\mathrm{C}$ and focal infiltrates of chronic inflammatory cells $\mathrm{D}$

(H\&E x 10)

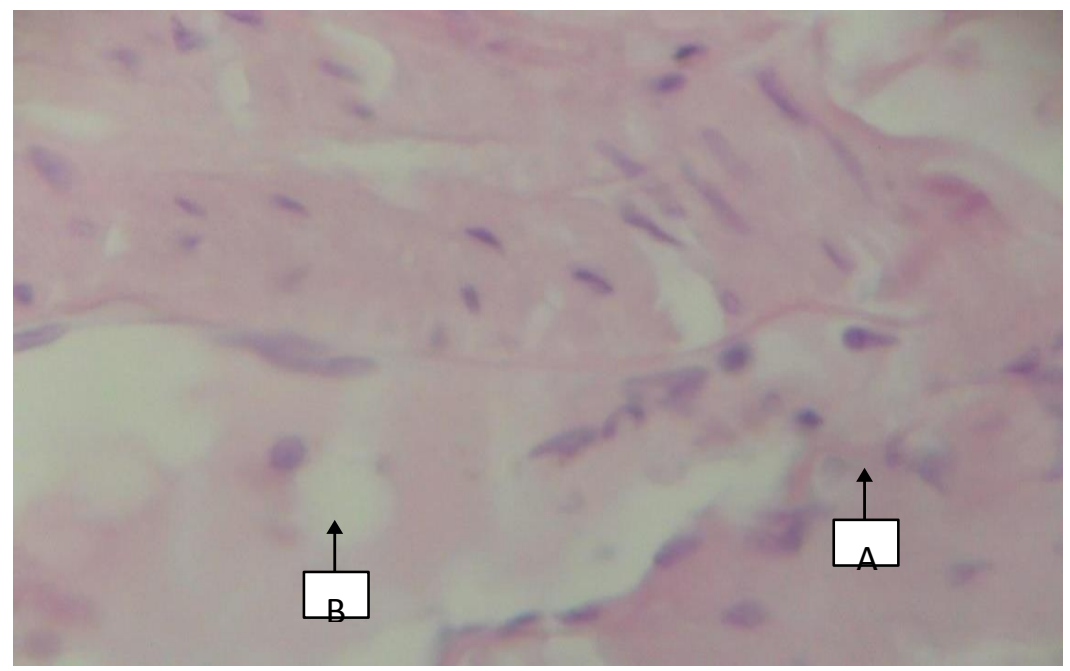

Plate 7: Higher power of the lesion above showing intimal ulceration A and deposition of hyalinised material B $(\mathrm{H} \& \mathrm{E} \times \mathrm{40})$

Group A: Is a slide section of the rabbit fed with normal food only, showing normal glomeruli, tubule and vasculatures of the kidney and also showing the normal inner lining of the blood vessels.

Group B: Is the group fed with normal food and instant noodle only, showing a moderate vascular congestion in the interstitial space. The $\mathrm{x} 40$ magnification of the group B shows chronic inflammation that is perivascular, mostly around the blood vessels. The glomeruli and tubules appear unremarkable.

Group C: Fed with normal food and seasoning only shows ulceration of the intima (inner lining of the blood vessels). The glomeruli and tubules appear unremarkable.

Group D: Fed with normal food and noodles with seasoning shows deposition of hyallinised materials inside the wall of the blood vessels and also, there is narrowing of the lumen of the blood vessels and deposition of the hyalinised materials. The glomeruli and tubules appear unremarkable.

\section{Discussion And Conclusion}

Several toxic substances ingested or eaten by the human being are eliminated by the combination of hepatic metabolism and renal excretion in the human, and kidney happens to be among the major organs of metabolism in the body (Brenner, 1999). Kidney plays a significant role in food breakdown. It's also important in drug excretorion in the body The noodlealone in plate 3 caused a chronic inflammation that is found around the blood vessels of the kidney, where has the seasoning alone caused damage to the inner lining of the blood 
vessel called intima which therefore confirmed the toxic nature of the seasoning if taken for a chronic duration to many organs. The combination of the noodle and the seasoning caused a greater damage to the wall of the blood vessels, thereby leading to the narrowing of the lumen of the blood vessels, almost blocking it, hence causing renal arteriosclerosis, which causes Ischaemia damage to the kidney. This Ischaemia damage to the kidney can lead to renal failure and increase in blood pressure (Hostetter, 2000). Additionally, the group that was given only seasoning plus feed showed a more severe effect on the kidney when compared with the other group. This outcome reasonably informed us that iflarge amount of seasoning is being added to noodle meal may be detrimental to the kindey.

\section{Conclusion And Recommendation}

The frequent consumption of instant noodles fast-food may affect the vital function of glomerular filtration, thereby resulting to morphological changes in the kidney followed by severe histopathologies.

This narrowing of the lumen of the kidney of blood vessels, thereby blocking it could cause renal arteriosclerosis, which cause Ischaemia damage to the kidney. It is recommended that further studies should be carried out to corroborate these findings.

\section{References}

[1]. Brenner BM, Rector FC Jr.(editors). The kindey, $6^{\text {th }}$ ed. 2 vols.

[2]. Chambille I, Serviere J, 1993, Neurotoxic effects of neonatal injections of monosodium L-glutamate on the retinal ganglion cell layer of the golden hamster: Anatomical and functional consequence on the circadian system. J comp Neurol.338(1):67-82.

[3]. Crosbie, G., S. Huang and J. Barclay. 1998. Wheat quality requirements of Asian foods. Euphytica 100: 155-156.

[4]. Drury RAB, Wallington EA, Cameron R.Carletons, 1967.Histological techniques: $4^{\text {th }}$ ed. Oxford university press NY. USA . 1967; 279-280.

[5]. Eweka A.O, Om Iniabors F.A.E. Histological Studies of the effects of monosodium Glutamate on the small intestine of Adult wistar rat. Electron J biomed 2007., 2:14-18.

[6]. Farombi E.O, Onyema O.O, 2006. Monosodium glutamate induced oxidative damage and genotoxicity in the rat: modulatory role of vitaminc, vitamin E, and quercetin. Hum ExpToxicol. 25(5):251- 9.

[7]. Hou G, 2001. Oriental noodles. Adv. Food Nutr, Res. 43:141-193.

[8]. Hostetter T.H, 2003. Prevention of the development and progression of renal disease. J AM socNephrol 14(suppl 2): S144.

[9]. Hou G and M. Kruk, 1998. Asian noodle technology. Technical bulletin 20 (12): 1 - 10.

[10]. Inagaki N, 1996. Regarding food poisoning caused by instant noodles. Food Sanit. Res. Jpn.16,

[11]. 370-379.

[12]. Kubomura. K. 1998, instant noodles in Japan. Cereal Hoods World 43:194-197. Macho L, Fickova M, Jezova, Zorad S, 2000. Late effects of postnatal administration of monosodium glutamate on insulin action in adult rats. Physiol Res. 49 suppl 1:579-85.

[13]. Morris, C. F., Jeifers, H, C. and Angele D. A, 2000. Effect of processing formulae

[14]. and measurement variables on alkaline noodle color-Toward an optimized laboratory system. Cereal Chem, 77:77-85.

[15]. Yun S. H, Quail K, and Moss R, 1996. Physicochemical properties of Australian wheat flours for white salted noodles. J. Cereal Sci. 23:181-189. D3. Accepted July 31,2003. 